auf der Suche nach Wirkungszusammenhängen befindet, ist dies überraschend. Insbesondere die sich aufdrängende Frage nach der Wirkungsweise der Reform von 1999, ihrer Effizienz und Zielgerichtetheit wird von Hansalek weder angesprochen noch beantwortet. Nur seine Einschätzung, der Reformprozess sei noch nicht abgeschlossen und weitere Schritte würden mit Sicherheit folgen, da diese „im Hinblick auf die Effizienzsteigerung der einzelnen Kontrolleinrichtungen sinnvoll“ (S. 7) seien, lässt den Leser erahnen, dass dieses Ziel durch die Reform von 1999 bislang nicht erreicht wurde.

Die Annahme, dass der beschriebene Reformprozess nicht abgeschlossen sei, dient zugleich als Begründung der Vorgehensweise, im ersten Teil zunächst die Empirie abzuhandeln, um sich im zweiten Teil allgemein-theoretischen Überlegungen zu widmen. Hansalek begründet dies mit dem Erfordernis, mit Blick auf mögliche weitere Reformen die parlamentarische Kontrolle der Bundesregierung im Bereich der Nachrichtendienste als Gesamtsystem zu erfassen. Auch dies wirft jedoch Fragen auf, da die hier erläuterten Grundlagen nicht in Bezug zur Reform von 1999 gesetzt werden. Da zudem keinerlei Schlussfolgerungen beziehungsweise konkrete Maßgaben für künftige Veränderungen aus den allgemeinen Überlegungen gezogen werden, hinterlässt auch dieser zweite Teil, der vergleichsweise abrupt mit einer stichwortartigen Aufzählung der Ergebnisse endet (für den ersten Teil wurde eine solche Zusammenfassung nicht erstellt), einen eher verwirrenden Eindruck beim Leser. So informativ das Buch hinsichtlich der Ausgestaltung des Kontrollsystems und seiner Reform ist, so sehr bleibt es hinter den Möglichkeiten weiterführender Analyse und der Herausarbeitung von Reformmaßstäben zurück.

Kristina Eichhorst

\title{
Entwicklung der Interparlamentarischen Union: verdienstvolle Dokumentation
}

Kissling, Claudia: Die Interparlamentarische Union im Wandel. Rechtspolitische Ansätze einer repräsentativ-parlamentarischen Gestaltung der Weltpolitik (Studien zum Öffentlichen Recht, Völker-und Europarecht, Bd.12), Peter Lang Verlag, Frankfurt am Main u.a. 2006, 736 Seiten, $€ 105,-$.

Bei einer Tagung von Parlamentariern aus neun Ländern wurde 1889 die Interparlamentarische Union (IPU) in Paris ins Leben gerufen. Wie Claudia Kissling in ihrer jüngst veröffentlichten juristischen Dissertation feststellt, ist die IPU damit die „erste politische internationale Organisation überhaupt“ (S. 29). Sie untersucht, ob und wie die IPU heute zu einer „parlamentarisch-repräsentativen Gestaltung der Weltpolitik“ (S. 30) beitragen kann. Im ersten Abschnitt ihrer historischen und rechtspolitischen Studie behandelt sie zunächst eingehend die ersten hundert Jahre der Organisation bis zum Fall der Berliner Mauer 1989. Mit dieser Gesamtbetrachtung trägt die Autorin dazu bei, eine seit langem vorhandene Forschungslücke zu schließen. In die Zeit bis 1914 fallen vor allem die Bemühungen der IPU um eine internationale Schiedsgerichtsbarkeit. In der Zeit nach dem Ersten Weltkrieg wurde versucht, den Völkerbund und die Weiterentwicklung des Völkerrechts zu unterstützen. Kissling schreibt, die IPU habe „die Entwicklung des Völkerbundes und später der 
Vereinten Nationen auf Regierungsebene maßgeblich beeinflusst“ (S. 29). Vom Zweiten Weltkrieg bis Anfang der 1990er Jahre verschwand die Organisation weitgehend von der politischen Bildfläche.

Basierend auf der Frage, welche Kriterien von Demokratie und Partizipation im Bereich der Global Governance bereits Eingang in das Völkerrecht gefunden haben, stellt Kissling im zweiten Abschnitt eine Vielzahl weiterer parlamentarischer Vereinigungen und Organe vor, denen sie eine gewohnheitsrechtliche Bedeutung zumisst. Die Anzahl und Relevanz dieser Institutionen, so wird festgestellt, sei besonders in den letzten Jahren stark gestiegen. Während 1939 nur die IPU, die Nordische IPU und die Parlamentarische Vereinigung des Commonwealth existierten, zählt Kissling heute rund 90 Vereinigungen dieser Art. Dabei haben sich viele strukturell nach dem Vorbild der IPU ausgerichtet. Allen sei gemein, dass sie danach streben „in einer veränderten, globalen Welt die Entscheidungen von Regierungsorganisationen auf internationaler Ebene zu kontrollieren, mitzugestalten und letztendlich durch Beteiligung von Volksvertretern zu legitimieren“ (S. 407). Neben dieser praktischen Betrachtung widmet sich die Autorin der Verankerung eines internationalen Demokratieprinzips auch im Vertragsrecht und im Soft Law. Dabei geht sie davon aus, dass „auch Ausführungen interparlamentarischer Versammlungen [...] aber auch nationaler Parlamente als Teil der Staatsgewalt im weitesten Sinne unter Soft Law gefasst werden können “ (S. 454). Tatsächlich fällt die Analyse des Vertragsrechts, wie etwa der UN-Charta, sehr sparsam aus und führt nur zu spärlichen Ergebnissen. Das untersuchte Soft Law ist jedoch, wie Kissling eingesteht, rechtlich unverbindlich und bietet nur Anhaltspunkte für eine politische Tendenz. Die Untersuchung zeigt allerdings, dass erste Elemente einer internationalen Demokratisierung auf dieser Ebene bereits entwickelt werden. Dies könnte sich letztlich auch auf die IPU auswirken.

Auf die Entwicklung der Union seit 1989 und die Ausbildung einer parlamentarischen Dimension bei der UNO konzentriert sich der dritte und letzte Abschnitt des Buches. Er stellt dar, wie die IPU im Zuge der fortschreitenden Globalisierung nach dem Ende des Kalten Krieges wieder an Kraft und Bedeutung gewonnen hat. Mit der Demokratisierungswelle in den 1990ern ging beispielsweise eine steigende Mitgliederzahl einher. Die IPU befasste sich außerdem intensiv mit zentralen politischen Fragen, etwa auf den Gebieten von Konfliktvorbeugung, Krisenmanagement, Rüstungskontrolle, Terrorismus und organisiertem Verbrechen, nachhaltiger Entwicklung oder Handels- und Wirtschaftsfragen. Die gewachsene Bedeutung spiegelt sich auch in der verstärkten Zusammenarbeit mit den Vereinten Nationen wider, die ausführlich dargelegt wird. So wurde 1996 ein Kooperationsabkommen geschlossen, 1997 wurde die IPU erstmals in ihrer Geschichte zu einer UN-Generalversammlung eingeladen, 2002 schließlich erhielt sie Beobachterstatus. Im Jahr 2000 fand am Rande des Millennium-Gipfels zudem erstmals eine viel beachtete Konferenz der Parlamentspräsidenten unter der Ägide der IPU statt. Kissling zeigt, wie im Zuge ihrer Revitalisierung auch eine Debatte über die zukünftige Entwicklung und Positionierung der IPU entstanden ist. Viele dort versammelte Parlamentarier sprachen sich dafür aus, die Union in eine Parlamentarische Versammlung bei der UNO umzuwandeln. Wie die Studie verdeutlicht, wurde die IPU teilweise schon 1912 als Vorläufer eines „Weltparlaments“ angesehen. In die Betrachtung sind auch Interviews von Kissling mit deutschen IPU-Delegationsmitgliedern und Verwaltungskräften 2002/2003 eingeflossen.

Zuletzt nimmt die Autorin zu den Aussichten und der Ausgestaltung eines „Gremiums zur Wahrnehmung der parlamentarischen Dimension bei der UNO“ (UNDP-Gremium) 
Stellung. Bezüglich einer verstärkten Einbeziehung nationaler Parlamentarier und Parlamente auf internationaler Ebene macht sie „in Fachkreisen eine Art Grundkonsens“ aus (S. 687). Als Gegenargumente identifiziert und diskutiert Kissling nichtsdestotrotz unter anderem die Politikverdrossenheit, die einem solchen Gremium die Basis raube; einen Mangel an Identifikation der Bürger mit der Welt; dass zunächst alle Staaten selbst Demokratien sein müssten und dass die UN-Mitgliedstaaten, ob nun demokratisch oder nicht, gar kein Interesse an einem solchen Gremium hätten. Unter bestimmten Voraussetzungen, so folgert Kissling, könne die IPU jedenfalls durchaus die Rolle eines UNDP-Gremiums einnehmen.

Das vorgelegte Werk ist für ein historisches Verständnis der IPU und als Hintergrundwissen für die Debatte um eine Parlamentarisierung auf internationaler Ebene sehr zu empfehlen. Unverzichtbar ist die Untersuchung für jeden, der sich speziell mit der IPU befasst. Sie ist allerdings mit 736 Seiten sehr umfangreich und enthält kein Register.

Besonders die im letzten Abschnitt behandelten Fragen sind zudem überaus aktuell. Im April 2007 haben sich beispielsweise mehrere hundert Persönlichkeiten aus über 80 Ländern, darunter knapp 400 Abgeordnete, in einem gemeinsamen Aufruf für die Einrichtung einer Parlamentarischen Versammlung bei den Vereinten Nationen ausgesprochen. Unter den Unterzeichnern sind auch zahlreiche Wissenschaftler, die sich mit der Frage internationaler Demokratie auseinandersetzen, wie etwa Daniele Archibugi, Richard Falk, Johan Galtung, David Held, Uwe Holtz, Lucio Levi, Heikki Patomäki, Andrew Strauss, Torbjörn Tännsjö oder Michael Zürn. Der Aufruf ist Kernstück einer internationalen Kampagne von Nichtregierungsorganisationen, die sich der Forderung nun verstärkt annehmen möchten.

Andreas Bummel

\section{Der Baden-Württembergische Landtag: große Fleißarbeit, unausgeschöpftes Erkenntnis- potential}

Eisele, Hansjörg: Landesparlamente - (k)ein Auslaufmodell? Eine Untersuchung zum deutschen Landesparlamentarismus am Beispiel des Landtags von Baden-Württemberg (Würzburger Universitätsschriften zu Geschichte und Politik, Band 8), Nomos Verlagsgesellschaft, Baden-Baden 2006, 406 Seiten, €69,-.

„Können Landtage durch ihre Arbeit noch die eigene Existenz rechtfertigen? Stimmen Theorie und Praxis überein?“ (S. 16) Oder sind Landesparlamente gar nur „dekoratives Relikt aus den Anfängen der Bundesrepublik Deutschland“? (S. 5) Diese Fragen will Hansjörg Eisele mit einer Untersuchung über den Landtag Baden-Württembergs beantworten. Aber er will noch mehr, denn: „Landesparlamente [werden] (...) auf absehbare Sicht ihre innere Existenzberechtigung verlieren (...), wenn sie nicht zu umfassenden, teilweise schmerzhaften Reformen bereit sind." (S. 5) Solche Reformmöglichkeiten streut der Autor immer wieder in seine Überlegungen ein.

Eiseles Untersuchung gliedert sich in vier Teile: Das erste Kapitel legt die theoretischen und methodischen Grundlagen offen, das zweite stellt den politischen und historischen 